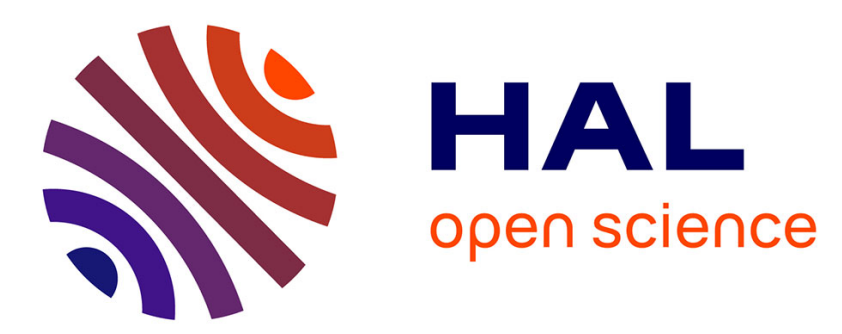

\title{
Intercarrier interference in uplink OFDMA systems with carrier frequency offset
}

\author{
Babar Aziz, Inbar Fijalkow, Myriam Ariaudo
}

\section{To cite this version:}

Babar Aziz, Inbar Fijalkow, Myriam Ariaudo. Intercarrier interference in uplink OFDMA systems with carrier frequency offset. IEEE 21st International Symposium on Personal Indoor and Mobile Radio Communications (PIMRC), 2010, Sep 2010, Istanbul, Turkey. pp.746 - 751, 10.1109/PIMRC.2010.5671922 . hal-00648519

\section{HAL Id: hal-00648519 \\ https://hal.science/hal-00648519}

Submitted on 5 Dec 2011

HAL is a multi-disciplinary open access archive for the deposit and dissemination of scientific research documents, whether they are published or not. The documents may come from teaching and research institutions in France or abroad, or from public or private research centers.
L'archive ouverte pluridisciplinaire HAL, est destinée au dépôt et à la diffusion de documents scientifiques de niveau recherche, publiés ou non, émanant des établissements d'enseignement et de recherche français ou étrangers, des laboratoires publics ou privés. 


\title{
Intercarrier Interference in Uplink OFDMA Systems with Carrier Frequency Offset
}

\author{
Babar Aziz, Inbar Fijalkow and Myriam Ariaudo \\ ETIS, ENSEA, Université de Cergy-Pontoise, CNRS, \\ F 95014 Cergy-Pontoise Cedex, France \\ E-mails: \{babar.aziz,inbar.fijalkow,myriam.ariaudo\}@ensea.fr
}

\begin{abstract}
Orthogonal Frequency Division Multiplexing (OFDM) is very sensitive to carrier frequency offsets (CFO). The CFO results in intercarrier interference (ICI). This drawback of OFDM is inherited to Orthogonal Frequency Division Multiple Access (OFDMA). In this paper, we propose a new ICI model for an uplink OFDMA system that takes into account the presence of the cyclic prefix which is usually ignored. Simulations validate the accuracy of our derivation. The subcarrier allocation is revisited in the presence of CFO.

Index Terms-Carrier Frequency Offset, Orthogonal Frequency Division Multiple Access, Cyclic Prefix.
\end{abstract}

\section{INTRODUCTION}

The upcoming mobile communication systems such as Wifi, WiMAX and LTE (Long Term Evolution) have to offer high data rates both in the uplink and downlink directions in order to fulfill the needs of the future innovative multimedia applications [1], [2]. OFDMA is coming forth as the favored downlink transmission scheme for these systems because it is highly adaptive and robust in frequency selective radio channels and also provides good system flexibility [1], [3]. However, questions are still raised about the use of OFDMA in the uplink direction. A well known problem of OFDMA in the uplink is its sensitivity to carrier frequency offsets (CFO). This paper concentrates on carrier frequency synchronization error while perfect time synchronization is assumed. CFO might result from Doppler shift, or in the case where a user mixer is not perfectly synchronized to its allocated carrier frequency [2], [4], [5]. CFO besides causing attenuation and rotation of each of the subcarrier, also causes loss of orthogonality between subcarriers leading to interference among adjacent subcarriers known as ICI. The main goal of this paper is to investigate the impact of ICI resulting from $\mathrm{CFO}$ in an uplink OFDMA system. Several researchers have studied the ICI due to changes in frequencies [6]-[10] in OFDM(A) systems. Analytical models of ICI provided in [7], [9] neglect the cyclic prefix which is in practice between $1 / 4$ and $1 / 8$ of the number of subcarriers. The cyclic prefix is inserted in an $\mathrm{OFDM}(\mathrm{A})$ symbol to cancel intersymbol interference and ICI in frequency selective channels. Some interesting results are provided in [8] but the ICI analysis is done using the Fourier Transform rather than DFT. So the results of [8] suit the case of an infinite number of carriers which is not very realistic.

\footnotetext{
${ }^{1}$ This work is supported by the French IdF Region DIM "Logiciels et Systmes Complexes" Project DESAP.
}

In this paper we provide an analytical model for ICI without neglecting the cyclic prefix. We will show that our analytical model is more accurate.

In section 2, the considered system model is presented along with an analytical model for the CFO depending ICI. In section 3 , we present the impact of CFO introduced multiuser interference through its variance analysis and simulation results. Section 4 discusses the CFO induced self-ICI and section5 introduces the effect of subcarrier allocation.

\section{UPLinK SYSTEM MODEL}

In this section, we present the uplink OFDMA system under consideration. The available bandwidth $B$ is divided into $N_{p}$ subcarriers. $N_{u}$ is the total number of users in the system. Each user is therefore allocated $M=N_{p} / N_{u}$ subcarriers exclusively to fulfill its transmission demands. The radio channel model is assumed to be frequency selective. The last $L$ modulation symbols in the OFDMA symbol are repeated after the inverse DFT to form the cyclic prefix.

\section{A. Analytical Signal Model}

We next describe the analytical signal model of the OFDMA system. The signal at the output of the transmitter of user $u$ is given by:

$$
x^{(u)}(t)=\operatorname{Re}\left\{e^{j 2 \pi\left(f_{c}+\delta f_{c}^{(u)}\right) t} \sum_{k=0}^{N_{p}-1} a_{k}^{(u)} p^{(u)}\left(t-\frac{k T}{N_{p}}\right)\right\}
$$

where $f_{c}$ is the carrier frequency, $p^{(u)}(t)$ is the impulse response of the low pass filter at the transmitter and $T$ is the symbol period of an OFDMA symbol. $\delta f_{c}^{(u)}$ represents the CFO of user $u$ with respect to $f_{c}$. The CFO appears in the transmitted signal because we are considering the case where the user $u$ is not perfectly synchronized to the carrier frequency of the base station. Note that $a_{k}^{(u)}$ are the symbols at the IDFT output given by:

$$
\underline{a}^{(u)}=\left(\begin{array}{c}
a_{N_{p}-1}^{(u)} \\
\vdots \\
a_{0}^{(u)}
\end{array}\right)=\underline{F}^{-1} \underline{\Pi}^{(u)} \underline{A}^{(u)}
$$

where $\underline{A}^{(u)}=\left\{A_{0}^{(u)} \ldots A_{M-1}^{(u)}\right\}$ are the input symbols from user $u$. $\underline{\Pi}^{(u)}$ is an $N_{p} \times M$ subcarrier mapping matrix for 
user $u . \underline{\Pi}_{k, p}^{(u)}$ with $k \in\left\{0, \ldots, N_{p}-1\right\}$ and $p \in\{0, \ldots, M-1\}$ models the multiple access scenario. It equals 1 for the carriers allocated to user $u$ and zero elsewhere. $F^{-1}$ is the DFT matrix given by

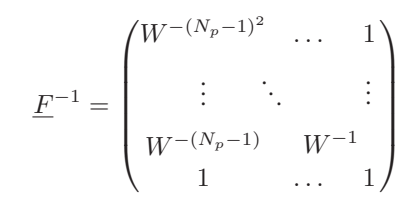

with $W=e^{j \frac{2 \pi}{N_{p}}}$. Signals from different users $x^{(u)}(t)$ pass through individual multipath radio channels. The received signal at the base station is the sum of the signals from all users. The overall noise free received signal $r(t)$ is given by:

$$
r(t)=\operatorname{Re}\left\{\sum_{u=1}^{N_{u}} \sum_{k=0}^{N_{p}-1} a_{k}^{(u)} q^{(u)}\left(t-\frac{k T}{N_{p}}\right) e^{j 2 \pi\left(f_{c}+\delta f_{c}^{(u)}\right) t}\right\}
$$

where $q^{(u)}(t)$ is the combined impulse response of the channel from user $u$ and of the transmitter and receiver filters. The baseband samples, at the symbol rate $T / N_{p}$, input to the receiver DFT can be written as:

$$
\left(\begin{array}{c}
r_{N_{p}-1} \\
\vdots \\
r_{0} \\
\vdots \\
r_{-L}
\end{array}\right)=\sum_{u=1}^{N_{u}}\left(\begin{array}{ccccc}
h_{o}^{(u)} & \cdots & h_{L-1}^{(u)} & & \\
\ddots & & \ddots & & 0 \\
& \ddots & & \ddots & \\
0 & & & & \\
& h_{o}^{(u)} & \cdots & h_{L-1}^{(u)}
\end{array}\right)\left(\begin{array}{ccc}
\delta^{\left(N_{p}+L-1\right)}(u) & \\
\ddots & & 0 \\
& \ddots & \\
0 & & \\
& & \delta^{0(u)}
\end{array}\right)\left(\begin{array}{c}
a_{N_{p}-1}^{(u)} \\
\vdots \\
a_{0}^{(u)} \\
\vdots \\
a_{-L}^{(u)}
\end{array}\right)
$$

with $L$ as the length of the cyclic prefix. Note that $\delta^{k(u)}=$ $e^{j 2 \pi k \delta f_{c}^{(u)} T / N_{p}}$ where $k=0, \ldots, N_{p}-1$ are the shift coefficients. The normalized shift of user $u$ is given by

$$
\delta f^{(u)}=\delta f_{c}^{(u)} T
$$

$r_{k}$ in equation (4) are the received symbols and $h_{k}^{(u)}$ is the discrete channel impulse response from user $u$ to the base station with $k=0, \ldots, L$. We assume that the channel impulse response length is smaller than $L$, so that the last terms of $h_{k}{ }^{(u)}$ can be zeros.

\section{B. Signal at the DFT output without CFO}

To analyse CFO induced ICI, we first recall the case of no CFO. When there is no CFO and each user is perfectly synchronized to the base station, the received signal is given by:

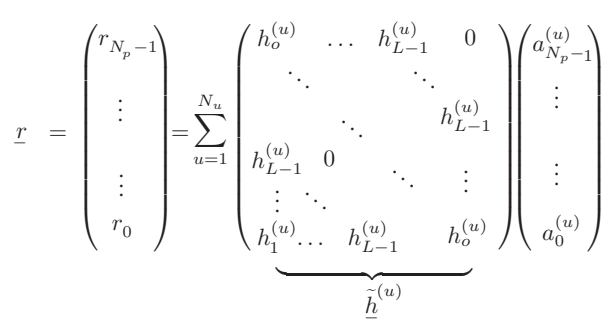

The last $L$ symbols of $\underline{r}$ in (4) corresponding to the cyclic prefix $a_{-1}, \ldots, a_{-L}$ have been discarded in (5) and the input symbols are rearranged since they are equal to $a_{N_{p}-1}, \ldots, a_{N_{p}-L}$. Thanks to the presence of the cyclic prefix, the channel matrix in (4) has been reduced to a circulant matrix $\underline{\widetilde{h}}^{(u)}$ in (5). This circulant channel matrix is further diagonalized by the receiver DFT. The received signal after the size $N_{p}$ DFT block is given by

$$
\underline{R}=\sum_{u=1}^{N_{u}} \underline{H}^{(u)} \underline{\Pi}^{(u)} \underline{A}^{(u)}
$$

where $\underline{H}^{(u)}$ is the diagonal channel matrix of user $u$ which is given by

$$
\underline{H}^{(u)}=\underline{F}_{\underline{\tilde{h}}^{(u)}}^{\underline{F}^{-1}}
$$

with entries $\underline{H}_{k, k}^{(u)}=\sum_{m=0}^{N_{p}-1} h_{m} e^{j 2 \pi m k / N_{p}}$. Knowing the allocation matrix $\underline{\Pi}^{(u)}$, the base station recovers all the input messages from $\underline{\Pi}^{(u)-1} \underline{R}$.

\section{Signal at the DFT output with CFO}

In the case of a CFO, $\delta f^{(u)}$ between a user $u$ and the receiver, we show next that the channel matrix doesn't remain circulant. If the receiver performs the usual cyclic prefix symbols discarding and rearrangement as when there is no $\mathrm{CFO}$, after some calculations, we get,

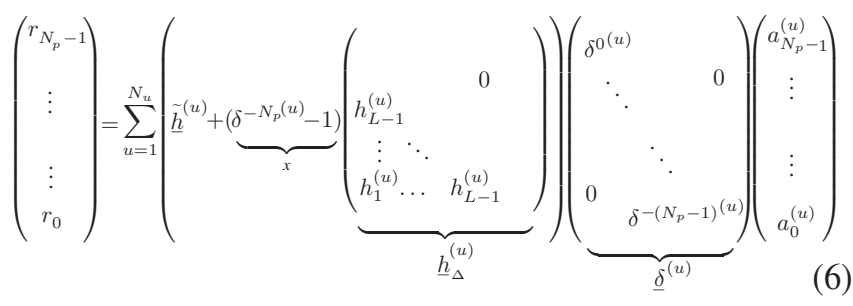

From equation (6), we find that the channel matrix can not be written in a circulant form unless we discard the additional terms $x \underline{h}_{\Delta}^{(u)}$ appearing in (6). Authors in [9], while analyzing the ICI due to CFO, have completely ignored the effect of CFO on the cyclic prefix in the received signal and the expression for the received signal is reduced to only

$$
\underline{R}=\sum_{u=1}^{N_{u}} \underline{H}^{(u)} \underline{\delta}^{(u)} \underline{a}^{(u)}
$$

Similarly the expressions for ICI in [2] or [9] correspond to the case where the effect of CFO is ignored on the cyclic prefix. The analytical expression we derive for ICI, $\zeta_{i c i}$, experienced by a particular user $v$ is given by

$$
\begin{aligned}
\underline{\zeta}_{i c i}^{(v)}=\sum_{u=1}^{N_{u}}\{ & \underline{H}^{(u)} \underline{F}\left(\underline{\delta}^{(u)}-\underline{I}\right) \underline{F}^{-1} \underline{\Pi}^{(u)} \underline{A}^{(u)} \\
& \left.+\left(\delta^{-N_{p}(u)}-1\right) \underline{F} \underline{h}_{\Delta}^{(u)} \underline{\delta}^{(u)} \underline{F}^{-1} \underline{\Pi}^{(u)} \underline{A}^{(u)}\right\}
\end{aligned}
$$

where $\underline{I}$ is an $N_{p} \times N_{p}$ identity matrix. Equation (7) represents 
the overall interference experienced by user $v$. It comes from multiple users interference as well as self-ICI.

\section{CFO Induced Multiple Users InTERFERENCE}

Next, we want to quantify the impact of multiple users interference by deriving its variance. For the sake of simplicity, we assume that there are only two users in the system, $N_{u}=2$. Furthermore, it is assumed that the base station is perfectly synchronized to user 1 i.e. $\delta f^{(1)}=0$ so that the interference comes from user 2 only $\delta f^{(2)} \neq 0$.

\section{A. Variance of ICI}

We assume in the sequel that the modulation symbols are statistically independent random variables from a predefined alphabet and independent from one user to another. Moreover, the transmit power for the modulation symbols is normalized such that $E\left\{\left|A^{(u)}\right|^{2}\right\}=1$. Then, the interference seen by the base station on the signal from user 1 due to the $\mathrm{CFO}$ in user 2 is reduced to

$$
\begin{aligned}
\underline{\zeta}_{i c i}^{(1)}=\underline{H}^{(2)} & \underline{F}\left(\underline{\delta}^{(2)}-\underline{I}\right) \underline{F}^{-1} \underline{\Pi}^{(2)} \underline{A}^{(2)} \\
& +\left(\delta^{-N_{p}^{(2)}}-1\right) \underline{F} \underline{h}_{\Delta}^{(2)} \underline{\delta}^{(2)} \underline{F}^{-1} \underline{\Pi}^{(2)} \underline{A}^{(2)}
\end{aligned}
$$

The total variance of (8) is given by equation (9),

$$
\sigma_{\zeta_{i c i}^{2}}^{2(1)}=\sigma_{1}^{2}+\sigma_{2}^{2}+\operatorname{cov}_{1,2}
$$

where $\sigma_{1}^{2}$ and $\sigma_{2}^{2}$ are the variances of the first and second terms of (8) respectively and $\operatorname{cov}_{1,2}$ represents the intercovariance of these terms. Since $F^{+}=F^{-1}$, the three terms of equation (9) are given by equations (10-12).

$$
\begin{aligned}
\sigma_{1}^{2}= & \operatorname{tr}\left\{\underline{F}^{-1} \underline{H}^{(2)} \underline{H}^{+(2)} \underline{F}\left(\underline{\delta}^{(2)}-\underline{I}\right) \underline{F}^{-1} \underline{\Pi}^{(2)} \underline{\Pi}^{+(2)} \underline{F}\left(\underline{\delta}^{(2)}-\underline{I}\right)^{+}\right\} \\
\sigma_{2}^{2}= & \left|\delta^{-N_{p}(2)}-1\right|^{2} \operatorname{tr}\left\{\underline{h}_{\Delta}^{+(2)} \underline{h}_{\Delta}^{(2)} \underline{\delta}^{(2)} \underline{F}^{-1} \underline{\Pi}^{(2)} \underline{\Pi}^{+(2)} \underline{F} \underline{\delta}^{(2)}\right\} \\
\operatorname{cov} v_{1,2}= & 2 \operatorname{Re}\left(\left(\delta^{-N_{p}(2)}-1\right)\right. \\
& \times \operatorname{tr}\left\{\underline{F} \underline{h}_{\Delta}^{(2)} \underline{\delta}^{(2)} \underline{F}^{-1} \underline{\Pi}^{(2)} \underline{\Pi}^{+(2)} \underline{F}\left(\underline{\delta}^{(2)}-\underline{I}^{+} \underline{F}^{-1} \underline{H}^{+(2)}\right\}\right)
\end{aligned}
$$

Note that in above equations $\operatorname{tr}(x)$ stands for the trace of $x$.

\section{B. Blockwise Case}

In this subsection, we consider that the subcarriers are allocated to the users in a block fashion as shown in Figure 1. In this case, the subcarrier allocation matrix of user 2 , $\underline{\Pi}^{(2)}$, can be simplified as

$$
\underline{\Pi}^{(2)}=[\underline{0} \underline{I}]^{T}
$$

where $\underline{0}$ is an $M \times M$ matrix with all entries equal to zero and
$\underline{I}$ is an $M \times M$ identity matrix. Thanks to this simplification and after some calculations we find that equations (10-12) reduce to:

$$
\begin{gathered}
\sigma_{1}^{2}=\sum_{k=0}^{M-1} \sum_{i=0}^{L-1} \sin ^{2}\left(\pi \delta f_{c}^{(2)} k T\right) h_{i}^{+(2)} h_{i}^{(2)} \\
\sigma_{2}^{2}=\left|\delta^{-N_{p}(2)}-1\right|^{2} \sum_{i=0}^{L-1} i\left(h_{i}^{+(2)} h_{i}^{(2)}\right) \\
\operatorname{cov}_{1,2}=2 \operatorname{Re}\left\{\left(\delta^{-N_{p}(2)}-1\right) \sum_{i=0}^{L-1} \sum_{k=1}^{i+1}\left(1-\delta^{-\left(N_{p}-k\right)(2)}\right) h_{i}^{+(2)} h_{i}^{(2)}\right\}
\end{gathered}
$$

Equations (10a-12a) represent the total variance of the interference seen by user 1 for a blockwise carrier allocation.

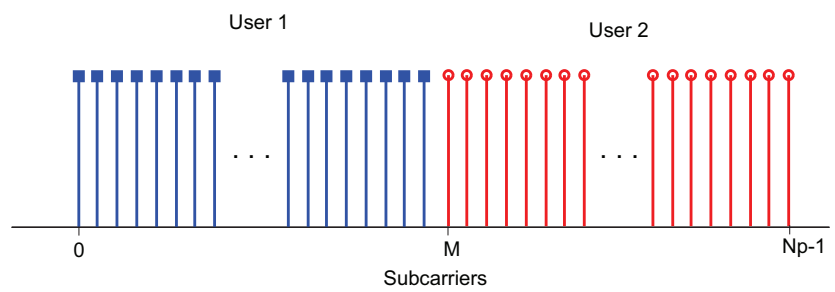

Fig. 1. Blockwise carrier allocation of user 1 and user 2. Each user is allocated $N_{p} / 2$ subcarriers that are adjacent to each other.

\section{Simulation Results}

Next, we compare the derived expression of the ICI with some previous results and with a simulated OFDMA system. For the simulations the following three scenarios are considered:

- Scenario 1(Sce.1): Simulated OFDMA transmission system with cyclic prefix. The corresponding ICI variance is denoted by $\sigma_{\text {sim }}^{2}$.

- Scenario 2(Sce.2): Based on CFO generated ICI model proposed in this paper. The corresponding ICI variance is denoted by $\sigma_{\zeta}^{2}$.

- Scenario 3(Sce.3): Based on CFO induced ICI model presented in [9]. The corresponding ICI variance is denoted by $\sigma_{n o-c p}^{2}$.

Here $N_{p}=64$, length of the cyclic prefix is $L=16, N_{u}=2$ therefore $M=32$ and the carriers are allocated using the blockwise allocation shown in Figure 1. Again we assume that the base station is synchronized to user 1 so that $\delta f^{(1)}=0$ and $\delta f^{(2)} \neq 0$. A 16-QAM modulation is used. Figure 2 shows the ICI variance versus the user 2 normalized shift values, $\delta f^{(2)}$. The variances have been averaged over all subcarriers for each value of the normalized shift. The results are also averaged over 5000 channel realizations, with random impulse responses of length $L$. Results in Figure 2 show that our proposed ICI variance for user $1, \sigma_{\zeta}^{2(1)}$, is much closer to the simulated ICI variance of user $1, \sigma_{\text {sim }}^{2(1)}$ than the one derived in 


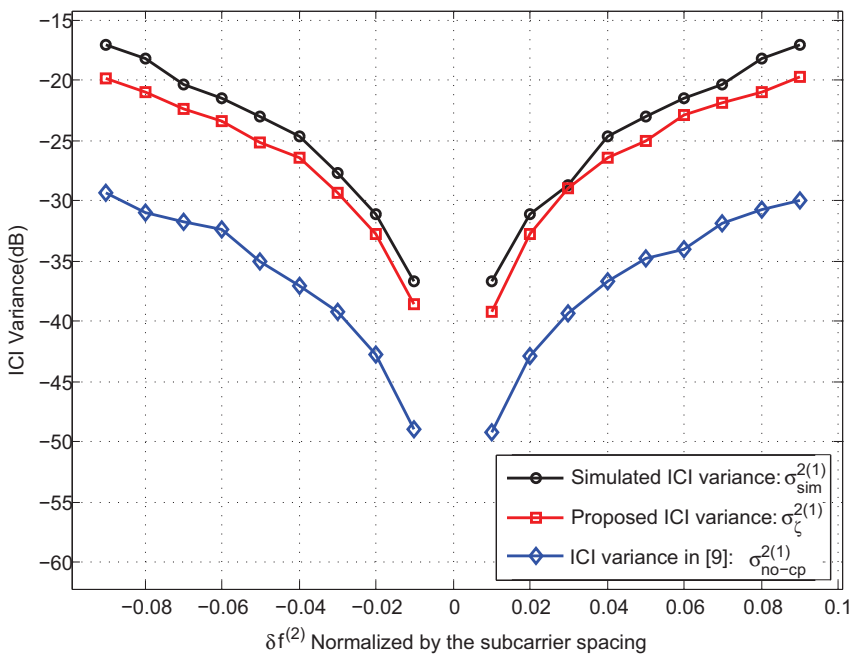

Fig. 2. Comparison of the ICI variances of user 1 for the three scenarios for different values of the normalized shift in user $2 \delta f^{(2)}$.

[9], $\sigma_{n o-c p}^{2(1)}$. At a shift of 0.08 the difference between $\sigma_{\text {sim }}^{2(1)}$ and $\sigma_{n o-c p}^{2(1)}$ is about $12 \mathrm{~dB}$. This result validates our proposed ICI model of multiple user interference and associated variance and shows its superiority with respect to the models in the literature.

\section{CFO InDUCED SELF-ICI}

In the previous section, we have assumed that the base station is synchronized to user 1 and we studied the multiple user interference in user 1 coming from user 2. Sticking to this assumption, we assume in this section that the base station can not be synchronized to user 2 which would have required a supplementary VCO. The interference seen by user 2 is therefore the interference from user 1 plus the interference coming from its own subcarriers, called self-ICI.

\section{A. Blockwise Case}

In the blockwise subcarrier allocation scheme of Figure 1, most subcarriers allocated to user 2, the ones in the second block, experience only self-ICI. Only the carriers on the edge experience interference from user 1 . The analytical expression of the variance can't be simplified any further, so that we don't recall it here. The following simulations consider the same setting as described previously. In Figure 3, the curves for ICI variance of user 2 are plotted against the normalized shifts. Again, we can see that our results are much closer to the simulated compared to the model in [9]. Moreover, we have plotted $\sigma_{\zeta}^{2(2)}$ for $L=0$ and it can be seen that it matches with $\sigma_{n o-c p}^{2(2)}$. In Figure 4, the curves for ICI variance of user 2 are plotted against its normalized shift for different lengths of the cyclic prefix, $N_{p}$ remains equal to 64 . We can see from Figure 4 that as the length of the cyclic prefix increases from $L=16$ to $L=22$ (about $\frac{1}{4} t h$ and $\frac{1}{3} r d$ of $N_{p}$ respectively), $\sigma_{\text {sim }}^{2(2)}$ and $\sigma_{\zeta}^{2(2)}$ increase accordingly. Indeed, $\sigma_{n o-c p}^{2(2)}$ remains constant. This result proves that considering our proposed model of ICI

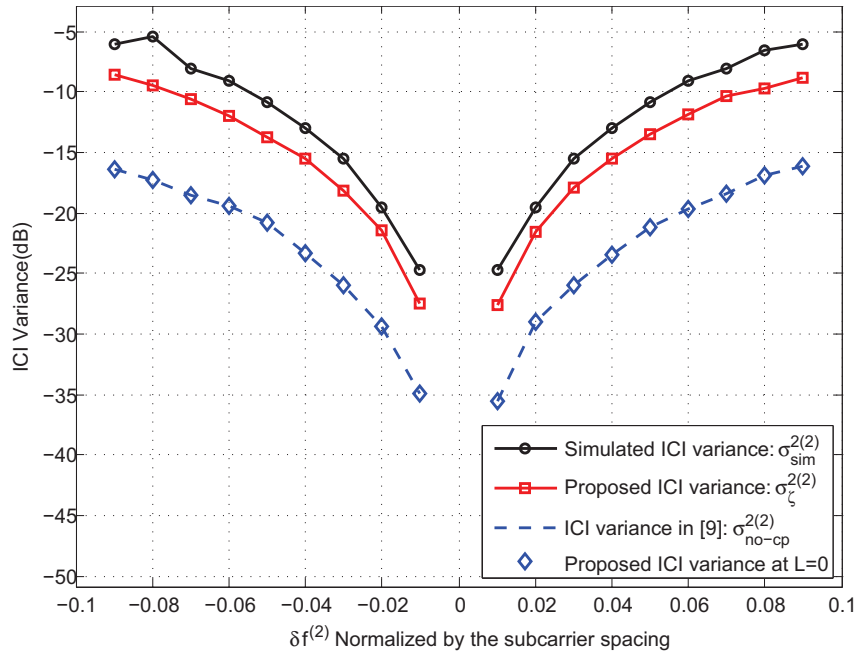

Fig. 3. Comparison of the ICI variances of user 2 for the three scenarios for different values of the normalized shift in user $2 \delta f^{(2)}$.

in OFDMA system with CFO is more important when the cyclic prefix is long with respect to the number of subcarriers.

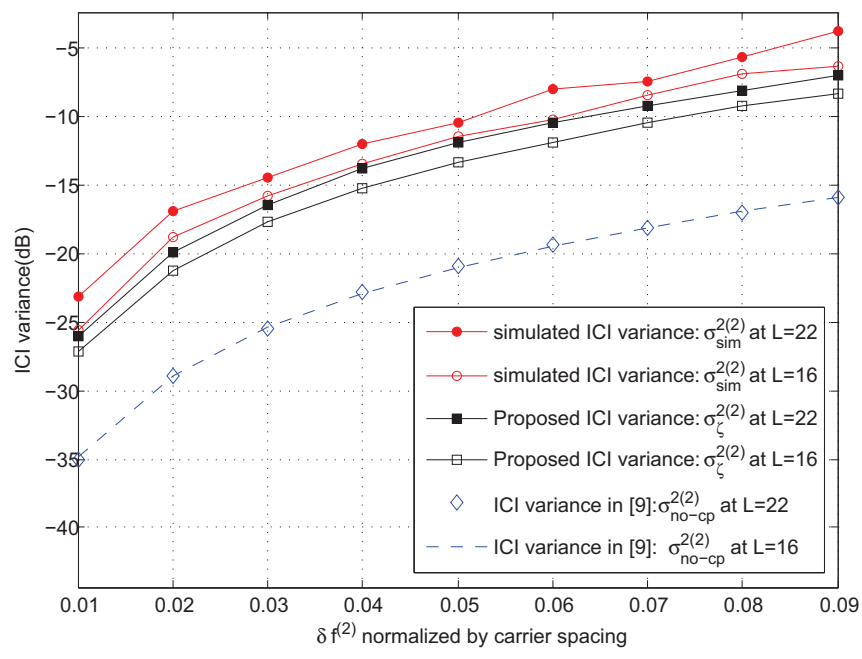

Fig. 4. Comparison of the ICI variances of user 2 for $L=16$ and $L=22$ for the three scenarios.

\section{B. Effect of ICI on Symbol Error Rate}

Next, we demonstrate the importance of the proposed model for the symbol error rate (SER) that is a better measure of performance than the variance of error. For the analytical SER calculation, we used the following formula [11]

$$
P_{s}=\frac{15}{16}-\sqrt{\frac{\gamma}{\gamma+10}}\left(\frac{3}{8}+\frac{9}{4 / \pi} \sqrt{\frac{\gamma}{\gamma+10}}\right)
$$

where $\gamma$ is the signal to interference plus noise ratio defined 


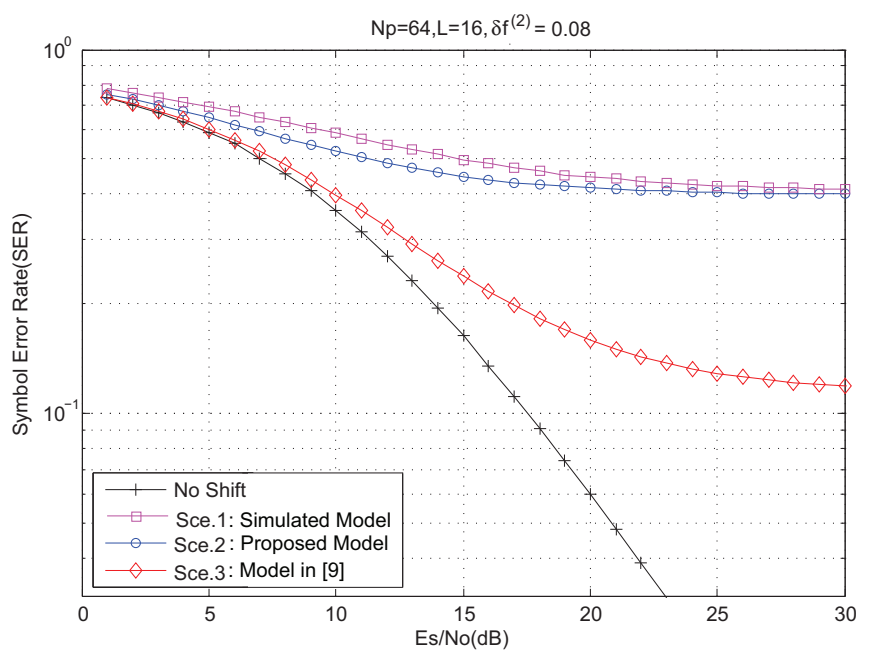

Fig. 5. Comparison of the SERs' of user 2 for the three scenarios at $N_{p}=64$ and $L=16$.

as $\gamma=E_{s} /\left(\sigma_{\zeta}^{2}+N_{0}\right)$ with $\sigma_{\zeta}^{2}$ as defined previously and $N_{0}$ is the noise power. Simulation results shown in Figure 5 give the SER performance of user 2 for the three scenarios at $\delta f^{(2)}=0.08$. Again $N_{p}=64$ and $L=16$. For such a high value of CFO, we can see that SERs of user 2 for Sce.1 and Sce. 2 are very close to each other, around $4.10^{-1}$ for high values of SNR while the SER for Sce.3 is much less i.e. close to $10^{-1}$. In Figure 6, the SER curves are plotted for different values of $L$. Indeed, the SER of user 2 for Sce.1 and 2 is higher at $L=22$ than at $L=16$. Again Sce. 3 shows no change in SER with the length of cyclic prefix since it ignores the cyclic prefix. These results validate our model of the SER in OFDMA systems with CFO and their superior accuracy with respect to the literature.

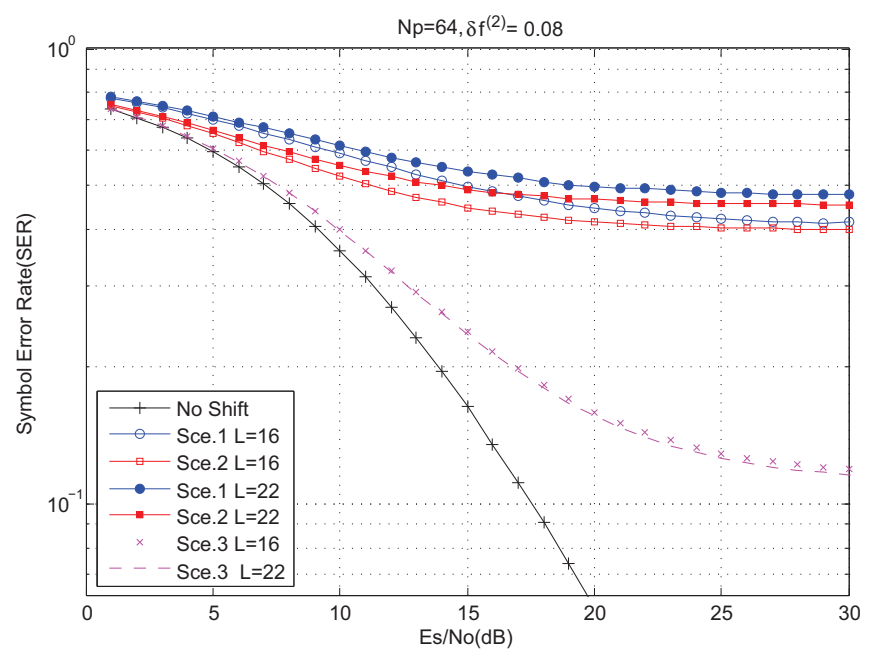

Fig. 6. Comparison of the SERs' user 2 for the three scenarios for different lengths of the cyclic prefix.

\section{ICI AND SUbCARRIER AllocATION}

In this section, we consider the impact of the subcarriers allocation on the ICI and related performance. In systems that do not undergo CFO, channel diversity is usually induced by interleaving the subcarriers allocated to the different users. However, in systems experiencing CFO a blockwise allocation minimizes the level of interference. It reduces the interference to the edge subcarriers of the block. Channel diversity and robustness to CFO are therefore in contradiction. In the sequel, we wonder what is the best number of adjacent subcarriers in the presence of CFO. The blockwise allocation scheme presented in Figure 1 corresponds to the maximum size a block can have, it is equal to $N_{p} / 2$.

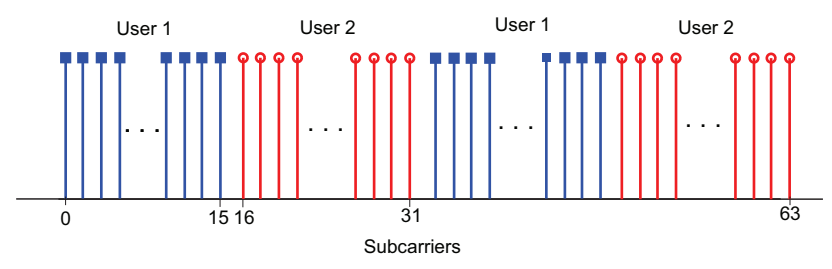

Fig. 7. Subcarrier allocation with blocksize equal to 16 .

The minimum will be equal to 1 , called the interleaved allocation. We simulate the same two user case presented in the previous sections with base station synchronized to user 1 while user 2 is not synchronized. The interference power is evaluated for user 1 and user 2 for blockwise allocation with different blocksizes (BS). A blockwise arrangement of the subcarriers for a blocksize of 16 is shown in Figure 7. Figure 8 shows the ICI variance for both users versus different values of blocksize (BS). $N_{p}=64, L=16$ and $\delta f^{(2)}=0.06$. At a blocksize of 32, the subcarriers of user 2 are far from

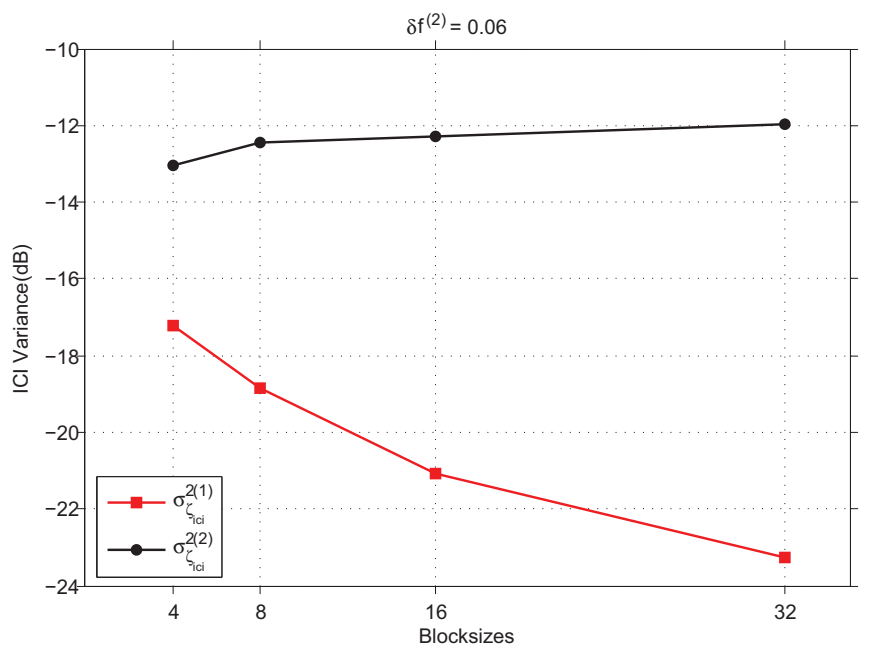

Fig. 8. ICI variances of the two users for different blocksizes at a normalized user 2 shift $\delta f^{(2)}=0.06$.

the subcarriers of user 1 (perfectly synchronized). So the 
interference seen by user 1 , with variance $\sigma_{\zeta}^{2(1)}$, because of $\mathrm{CFO}$ in user 2 is only at the edge subcarriers and is small i.e. less than $-23 \mathrm{~dB}$. While the ICI in user 2 , with variance $\sigma_{\zeta}^{2(2)}$, is high $-12 \mathrm{~dB}$ and is caused by its own subcarriers. Figure

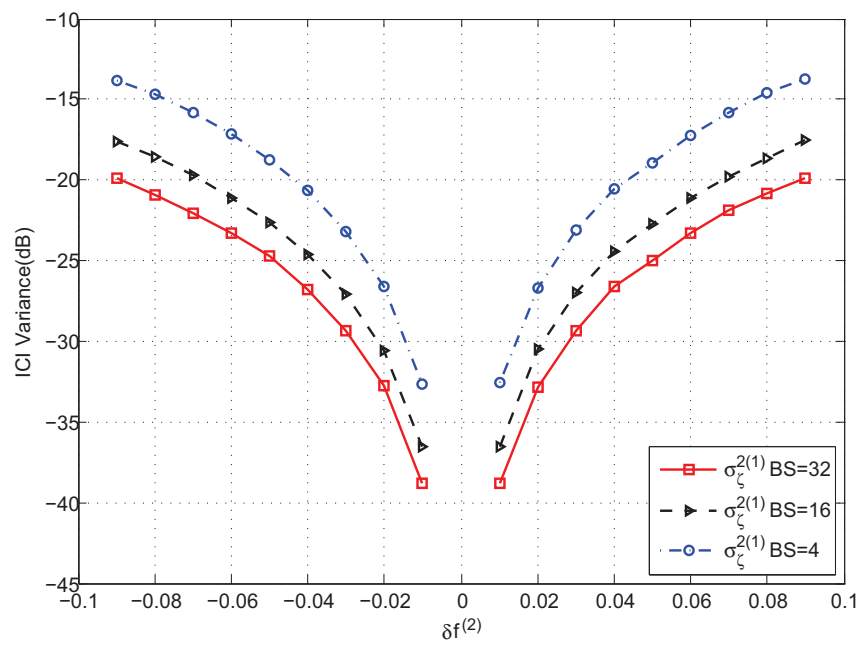

Fig. 9. ICI variance of user 1 for different values of $\delta f^{(2)}$ with blocksizes 32, 16 and 4 .

8 shows that with a decreasing blocksize, $\sigma_{\zeta}^{2(1)}$ increases while $\sigma_{\zeta}^{2(2)}$ decreases. This is because the only interference user 2 sees is from its own carriers. With a decreasing blocksize, user 2 subcarriers become far from each other and become closer and closer to user 1 subcarriers which offer no interference. In Figure 9, the ICI variance for user 1 is detailed for different values of normalized shift $\delta f^{(2)}$ for blocksizes 32,16 and 4 . We can see that with the increase in blocksize the ICI power for user 1 decreases. As expected, a larger blocksize provides more robustness against CFO. However, the gain in diversity does not seem to compensate the loss due to CFO for small values of CFO.

\section{CONCLUSION}

A new analysis of the effect of carrier frequency offset (CFO) in an uplink OFDMA systems has been presented. We have demonstrated that one must take into account the cyclic prefix while analyzing Intercarrier Interference (ICI) resulting from CFO. We proposed an analytical expression of the ICI, the associated variance and SER. It allows us to study the subcarrier allocation for an OFDMA system undergoing CFO.

\section{REFERENCES}

[1] A. F. Molisch, Wideband Wireless Digital Communication, Upper Saddle River: Prentice Hall, 2001.

[2] Qinfei Huang, Mounir Ghogho, Jibo Wei, and Philippe Ciblat, "Timing and frequency synchronization for OFDM based cooperative systems," ICASSP, IEEE International Conference on Acoustics, Speech and Signal Processing, pp. 2649-2652, 2009.

[3] Galda D. Gruenheid R Rohling, H., "OFDM:A flexible and adaptive air interface for a $4 \mathrm{G}$ mobile communication system," International Conference on Telecommunications(ICT), Beijing, pp. 5-14, 2002.

[4] P. H. Moose, "A technique for orthogonal frequency division multiplexing frequency offset correction," IEEE Transactions on Communications, vol. 42, no. 7, pp. 2908-2914, Oct. 1994.

[5] H. Sari, G. Karam, and I. Jeanclaude, "Channel equalization and carrier synchronization in OFDM systems," in presented at 1993 Tirrenia Int.Workshop on Digital Communications, Tirrenia, Italy, September 1993.

[6] J. Armstrong, "Analysis of New and Existing Methods of Reducing Intercarrier Interference due to Carrier Frequency Offset in OFDM," IEEE Transactions on Communications, vol. 47, pp. 27-32, July 2001.

[7] Stefan Kaiser Patrick Robertson, "Analysis of Doppler Spread Perturbations in OFDM(A) Systems," IEEE Transactions on Communications, vol. 11, no. 6, November-December 2000.

[8] Leonard J. Cimini Ye (Geoffrey) Li, "Bounds on the Interchannel Interference of OFDM in Time-Varying Impairments," IEEE Transactions on Communications, vol. 49, no. 3, March 2001.

[9] Martin Stemick and Hermann Rohling, "Effect of Carrier Frequency Offset on the Channel Capacity in Multiuser OFDM-FDMA Systems," Wireless Personal Communications: An international Journal, vol. 47 no. 1, pp. 5-14, Oct. 2008.

[10] Yuping Zhao and Sven-Gustav Haggman, "Intercarrier interference selfcancellation scheme for OFDM mobile communication systems," IEEE Transactions on Communications, vol. 49, no. 7, pp. 1185-1191, July 2001.

[11] J.P.M.G. Linnartz Editor-in Chief, Wireless Communication, The Interactive Multimedia CDROM, Baltzer Science Publishers / Kluwer Ac. P., ISSN 13834231. 